\title{
A NOTE ON THE TIME-NON-HOMOGENEOUS JOHNSON-MEHL TESSELLATION
}

\author{
VRATISLAV HORÁLEK, ${ }^{*}$ National Research Institute for Machine Design, Praha
}

\begin{abstract}
The main characteristics of the time-nonhomogeneous Johnson-Mehl tessellation with specified nucleation intensity $\alpha(t)=\alpha t^{\beta}, \beta>-1$ and $\alpha>0$ being constants and $t$ time, are investigated as functions of $\beta$.
\end{abstract}

POISSON-VORONOI TESSELLATION; JOHNSON-MEHL TESSELLATION

Miles (1972) presented a review of models concerning the random division of the space $\mathbb{R}^{3}$ into space-filling and non-overlapping cells. In this note attention is focused on three of these models: (i) the Poisson-Voronoi (PV) tessellation, (ii) the time-homogeneous Johnson-Mehl (THJM) tessellation, (iii) the time-non-homogeneous Johnson-Mehl (TNHJM) tessellation. The PV tessellation (i) has convex cells, whereas the JM tessellations (ii) and (iii) have non-convex cells. The purpose of this note is to extend the results derived by Miles (1972) for the TNHJM tessellation and to show some internal relationships between the PV tessellation and the TNHJM tessellation with nucleation intensity

$$
\alpha(t)=\alpha t^{\beta},
$$

$\beta>-1$ and $\alpha>0$ being constants and $t$ time. As in Miles's paper we shall consider the 'ergodic' moments of the volume $V$, the surface area $S$, the total edge length $L_{1}$ and the number of vertices $N_{0}$ of the 'typical cell'.

Inserting (1) into the expressions (69)-(74) of Miles's paper, all integrals involved there convert into gamma functions. Hence for the TNHJM tessellation with intensity (1) we have that

$$
\begin{gathered}
E(V)=k_{V}(\beta)\left\{\frac{v^{3(\beta+1)}}{\alpha^{3}}\right\}^{1 /(\beta+4)}, \\
E(S)=k_{S}(\beta) N_{V}^{-\frac{2}{3}} ; \quad E\left(L_{1}\right)=k_{L_{1}}(\beta) N_{V}^{-\frac{1}{3}} ; \quad E\left(N_{0}\right)=k_{N_{0}}(\beta),
\end{gathered}
$$

where

$$
\begin{gathered}
k_{v}(\beta)=\frac{1}{\Gamma\left(\frac{\beta+1}{\beta+4}\right)}\left\{\frac{2^{3(\beta+1)} \pi^{\beta+1}(\beta+4)^{3}}{[(\beta+1)(\beta+2)(\beta+3)]^{\beta+1}}\right\}^{1 /(\beta+4)}, \\
k_{S}(\beta)=\frac{8}{3} \frac{\Gamma\left(\frac{2 \beta+7}{\beta+4}\right)}{\left\{\Gamma\left(\frac{\beta+1}{\beta+4}\right)\right\}^{\frac{1}{3}}} \frac{\pi^{\frac{1}{3}}(\beta+4)}{\{(\beta+1)(\beta+2)(\beta+3)\}^{\frac{1}{3}}},
\end{gathered}
$$

Received 1 February 1988; revision received 17 May 1988.

* Postal address: National Research Institute for Machine Design, 19011 Praha 9-Bèchovice II, Czechoslovakia. 


$$
\begin{aligned}
& k_{L_{1}}(\beta)=\frac{4}{5} \frac{\Gamma\left(\frac{3 \beta+10}{\beta+4}\right)}{\left\{\Gamma\left(\frac{\beta+1}{\beta+4}\right)\right\}^{\frac{2}{3}}} \frac{\pi^{\frac{5}{3}}(\beta+4)^{2}}{\{(\beta+1)(\beta+2)(\beta+3)\}^{\frac{2}{3}}}, \\
& k_{N_{0}}(\beta)=\frac{32}{105} \frac{\Gamma\left(\frac{4 \beta+13}{\beta+4}\right)}{\Gamma\left(\frac{\beta+1}{\beta+4}\right)} \frac{\pi^{2}(\beta+4)^{3}}{(\beta+1)(\beta+2)(\beta+3)},
\end{aligned}
$$

$N_{V}=1 / E(V)$ is the mean number of cells per unit volume and $v$ constant growth rate, the growth being isotropic.

The numerical values of the coefficients $k_{i}(\beta), i=V, S, L_{1}, N_{0}$, for chosen values of $\beta \in\langle-1 ; \infty\rangle$ are summarized in Table 1 . Of course, for $\beta=0$ the TNHJM tessellation with intensity (1) converts into the THJM tessellation with constant intensity $\alpha$ and the corresponding coefficients $k_{i}(\beta)$ in Table 1 agree with those derived in Meijering (1953), Gilbert (1962) and Miles (1972). The limits of $k_{s}(\beta), k_{L_{1}}(\beta)$ and $k_{N_{0}}(\beta)$ for $\beta$ tending to -1 coincide with the corresponding coefficients of the PV tessellation (see the three papers mentioned above), for which it holds that $E(V)=1 / \alpha$. Note that the term in brackets in (2) converges to $1 / \alpha$ as $\beta$ tends to -1 . Furthermore, the coefficients $k_{i}(\beta), i=S, L_{1}, N_{0}$, are decreasing functions in the interval $\langle-1 ; \infty)$ and have finite positive limits for $\beta \rightarrow \infty$. Finally, the coefficient $k_{V}(\beta)$ is a unimodal function having its maximum 1.2647 at the point $\beta=0.56$ and it converges to 0 when $\beta$ tends to -1 and to $\infty$.

TABLE 1

Coefficients $k_{i}(\beta), i=V, S, L_{1}, N_{0}$

\begin{tabular}{lllll}
\hline \multicolumn{1}{c}{$\beta$} & $k_{V}(\beta)$ & $k_{S}(\beta)$ & $k_{L_{1}}(\beta)$ & $k_{N_{0}}(\beta)$ \\
\hline-1.0 & 0 & 5.8209 & 17.4956 & 27.0709 \\
-0.75 & 0.3128 & 5.5808 & 16.4487 & 25.2662 \\
-0.50 & 0.6475 & 5.4005 & 15.7075 & 24.0630 \\
-0.25 & 0.9245 & 5.2587 & 15.1506 & 23.2036 \\
0 & 1.1161 & 5.1433 & 14.7143 & 22.5591 \\
0.25 & 1.2241 & 5.0473 & 14.3621 & 22.0578 \\
0.50 & 1.2635 & 4.9659 & 14.0708 & 21.6567 \\
0.75 & 1.2520 & 4.8959 & 13.8253 & 21.3286 \\
1.0 & 1.2062 & 4.8348 & 13.6155 & 21.0552 \\
2.0 & 0.8944 & 4.6526 & 13.0058 & 20.3032 \\
$\infty$ & 0 & 3.9056 & 10.7821 & 18.0473 \\
\hline
\end{tabular}

\section{References}

Gilbert, E. N. (1962) Random subdivision of space into crystals. Ann. Math. Statist. 33, 958-972.

MEIJERING, J. L. (1953) Interface area, edge length and number of vertices in crystal aggregates with random nucleation. Philips Res. Report 8, 270-290.

Miles, R. E. (1972) The random division of space. Proc. Symp. Statist. and Probab. Problems in Metallurgy, Adv. in Appl. Probability, Special Suppl., 243-266. 